\title{
ELECTRIC POLARIZATION OF ONSAGER FLUIDS. I. DIPOLE POLARIZATION. 3. THE ROLE OF UNIVERSAL PAIRWISE INTERACTIONS
}

\author{
V.V. Prezhdo ${ }^{a}$, G.V. Tarasova ${ }^{b}$, O.V. Prezhdo ${ }^{c}$, S.A. Tyurin ${ }^{b}$, \\ N.I. IVANOV ${ }^{b}$ AND T.N. KURSKaYA ${ }^{b}$ \\ ${ }^{a}$ Institute of Chemistry, University of Opole, Oleska 48, 49-951 Opole, Poland \\ ${ }^{b}$ Ukrainian Scientific Center for Water Control, Kharkov, Ukraine \\ ${ }^{c}$ Department of Chemistry, University of Texas, Austin, USA
}

(Received March 7, 1995; in final form May 9, 1995)

\begin{abstract}
A comparative study of the methods of interpretation of solvent influences on electric properties of molecules was conducted based on experimental dielectric properties of dilute solutions of organic substances of various polarity. It was shown that the solvent effect estimated via London-Debye-Keesom pairwise interaction potentials is best accounted for by theories of polarization of condense media which develop the anzatzes of reactive field and local dielectric permittivity.
\end{abstract}

PACS numbers: 35.20.My, 31.70.Dk

\section{Introduction}

The dipole moment of a molecule in a condensed phase (condensate) $\mu_{\mathrm{c}}$ differs from the dipole of a free molecule $\mu_{0}$ by the contribution $\mu_{\text {ind }}$ induced by the electric field $E(\tau)$ of $N$ neighboring molecules

$$
\mu_{\mathrm{c}}=\mu_{0}+\mu_{\text {ind }}=\mu_{0}+N \frac{\int \alpha E(\tau) \exp (-W / k T) \mathrm{d} \tau}{\int \exp (-W / k T) \mathrm{d} \tau},
$$

where $\mathrm{d} \tau$ is an element of the configuration space which characterizes the position and orientation of the molecule with dynamic polarizability $\alpha ; W$ is the total potential energy of the particle, consisting of the potential energy of the molecule in the external field $E$ produced by distant neighbors and the energy of interparticle interactions with the nearer ones $U_{\mathrm{N}}: W=U_{\mathrm{N}}-\mu_{0} E$. Unfortunately, there exists no universal practical scheme which would allow for the calculation of all contributions to $W$. This still remains the central and the most difficult goal of condensed phase physics. On the one hand, it is possible to study the polarizability of polar 
media by statistical physics techniques, and, thus in principle to determine the nature and extent of the interparticle interactions influencing dielectric properties. Well-known statistical theories of Kirkwood-Fröhlich, Harris-Older, and Buckingham introduce empirical potentials to model the nearest order dipole-dipole couplings and give a general approach to such studies. On the other hand, in spite of all the advantages inherent consistency, rigorous theoretical foundations, there is necessity to know only microscopic parameters of these methods as well as the quantum mechanical ones are not applicable to the majority of practically relevant cases. It is clear that without reliable structural information the values of $E$ and $W$, and, hence, $\mu_{\mathrm{c}}$ cannot be accurately calculated. This complexity inevitably leads to approximation schemes incorporating various models. Although continuum theories are limited a priori in the sense that they do not consider interparticle interactions explicitly, they can be thought of as a first approximation to the exact description of the polarization of polar fluids. In the Onsager model of the internal field $E_{\mathrm{e}}$ and its components, the cavity field $G$ and the reactive field $R$, the energy $W$ in (1) is set equal to the potential energy of a molecule in the field $E_{\mathrm{e}}$. Based on this assumption a straightforward analytic relationship between $\mu_{0}$ and micro- and macroscopic parameters of a medium is derived as a solution to a typical boundary problem in the electrostatics of dielectrics and leads to the direct comparison of theory with experimental results.

Rigorous determination of the potential energy of interacting particles is a complicated many-body problem. It has been solved exactly for very few cases. The vast majority of computations assume additivity of independent electrostatic energies of pairwise interactions, though it is true only for Coulomb fields of single atoms. Empirical potentials used in such computations are analogous to the orientationally averaged adiabatic quantum mechanical potentials of electrostatically interacting particles calculated by various perturbation theories leading to the multipole expansion in a series of powers of $1 / r$ under the assumption of non-overlapping charge distributions. In first order, the energy of multipole couplings is determined by the unretarded pairwise electrostatic van-der-Waals interactions (Keesom-Rayganum forces) omitting the Kazimir-Polder retardation effect. In second order, the Debye-Falkenhagen-Margenau inductive and dispersive forces describe the polarization energy. The first term in the expansion of this energy is the well-known London-Margenau relation, which expresses the dispersion constant $C_{6}$ as a function of dynamic polarizabilities and first ionization potentials $I_{1}$. These would be substituted for effective oscillator strengths in a quantum mechanical formalism. Let us note here that the London formula gives the lower limit of the dispersion interaction energy, while the upper limit can be calculated within the limits of the Slater-Kirkwood approach.

As widely presented in the literature, qualitative and quantitative estimates of the influence of interparticle van-der-Waals interactions on resonance, spectral, thermodynamic and other characteristics of gases, carried out under the approximation of pairwise couplings, do agree well with experimental data. A similar treatment of condensed phases encounters substantial difficulties, the most crucial one being the necessity to include higher-order perturbations to account adequately for nonadditivity of many-particle interactions. Higher-order terms in 
the multipole expansion terms are complicated many-variable functions with limited applicability in analytical approaches. Computational experiments, such as numerical Monte Carlo and molecular dynamics simulations with semi-empirical atom-atom potentials, can be applied to systems with a relatively small number of particles $\left(10^{3}\right.$ compared to the Avogadro number $\left.10^{23}\right)$.

We undertook to establish relationships between the interparticle interactions in a condensate and its polarization properties. We used various continuum theories of liquid media to estimate gaseous phase dipole moments of solutes on the basis of dielectric properties measurements on binary solutions of organic compounds and then compared calculated dipole moments with the experimental gaseous phase values.

\section{Interparticle pairwise interactions and dipole polarization in solutions}

To define the gaseous phase electric dipole moment of a solute molecule we follow our earlier paper [1]. According to it the Fröhlich moment $M_{0}$ (which is the dipole moment of a polarized sphere of volume $V_{0}$ in a densely packed fluid medium) due to the external field $E_{0}$ is calculated on a macroscopic level involving the refraction index $n$ of the medium

$$
\left(\varepsilon-n^{2}\right) V_{0} E_{0}=4 \pi M_{0}
$$

where $\varepsilon$ is the dielectric constant of the medium. To describe a binary solution we introduce the notation: $x_{i}$ are mole fractions, $\rho_{i}$ and $M_{i}$ are densities and molecular weights, and $i=0,1,2$ denote solution, solvent and solute respectively. In a reasonably dilute binary solution ( $x_{2}$ is less than 0.01 ) without substantial concentrational fluctuations the first solvation shells surrounding a solute molecule include only solvent molecules. The following formulas hold:

$$
\rho_{0}=\rho_{1}\left(1+\beta x_{2}\right), \quad \varepsilon_{0}=\varepsilon_{1}\left(1+\delta x_{2}\right), \quad n_{0}=n_{1}\left(1+\gamma x_{2}\right) .
$$

In accordance with [1] the gaseous phase dipole moment of a solute molecule $(i=2)$ can be deduced from the dielectric characteristics of the solution and its components

$$
A \mu_{2}^{2}=\left\{\begin{array}{lll}
B(C+D-F), & \text { when } & \mu_{1}=0, \\
B(C-K)+L(K+D), & \text { when } & \mu_{1} \neq 0,
\end{array}\right.
$$

where

$$
\begin{aligned}
& A=\frac{4 \pi N_{\mathrm{A}}}{3 k_{\mathrm{B}} T M_{2}} \frac{1+1.5 \alpha_{2} t_{1}}{\left(1-\alpha_{2} t_{1}\right)^{3}}, \quad B=\frac{\varepsilon_{1}-1}{\rho_{1} g_{1}}, \\
& C=1-\beta-\frac{\mathrm{d}}{\mathrm{d} x_{2}}\left(\ln g_{0}\right)_{x_{2}=0}+\frac{\delta \varepsilon_{1}}{\varepsilon_{1}-1} \\
& D=\frac{M_{2} \rho_{1}}{M_{1} \rho_{2}}, \quad F=\frac{1}{1-\alpha_{1} t_{1}} \frac{\mathrm{d}}{\mathrm{d} x_{2}}\left(\alpha_{1} t_{0}\right)_{x=0}, \\
& K=\frac{3\left(1.5+\alpha_{1} t_{1}\right)}{\left(1-\alpha_{1} t_{1}\right)\left(1+1.5 \alpha_{1} t_{1}\right)} \frac{\mathrm{d}}{\mathrm{d} x_{2}}\left(\alpha_{1} t_{0}\right)_{x_{2}=0}
\end{aligned}
$$




$$
L=\frac{3\left(n_{1}^{2}-1\right)}{\left(n_{1}^{2}+2\right)\left(1-\alpha_{1} t_{1}\right) \rho_{1}} .
$$

$N_{\mathrm{A}}, k_{\mathrm{B}}$ and $T$ are Avogadro's number, Boltzmann's constant and absolute temperature, respectively. The expressions for the cavity and reactive field factors $g_{i}$ and $t_{i}$ and their derivatives with respect to the concentration of the solute (in the limit of $x_{2}=0$ ) are given in [2]. Reference [3] discusses the expression for the Onsager volumes: $a_{i}=r_{i}^{*}(0.86 \pm 0.03)^{1 / 3}$, where $r_{i}^{*}$ are the van-der-Waals radii of solvent and solute molecules.

It was shown in [4] that the potential energy of interparticle van-der-Waals interactions in a dense molecular medium can be approximated by a sum of the energies due to pairwise long range forces of dipole, inductive and dispersive origins. The expected systematic influence of the interactions on the calculated values of $\mu_{2}$ is estimated by means of the corresponding London-Debye-Keesom (LDK) potentials. For this purpose we express the molecular interaction energy averaged over spacial configurations energy in a binary solution of polar components as [5]:

$$
\begin{aligned}
& \left\langle U_{12}\right\rangle_{\tau}=\sum_{m}\left(\frac{\bar{Z}}{\bar{R}^{6}}\right)_{m}\left\langle\delta_{\mathrm{dis}} \Phi_{\mathrm{L}}+\delta_{\text {ind }} \Phi_{\mathrm{D}}+\delta_{\mathrm{dip}} \Phi_{\mathrm{K}}\right\rangle_{\tau} \\
& =A \sum_{m}\left(\frac{\bar{Z}}{\bar{R}^{6}}\right)_{m}\left[\alpha_{1}\left(1.5 I_{11}+I_{12} \alpha_{2}+\mu_{2}^{2}\right)+\mu_{1}^{2}\left(\alpha_{2}+\frac{2 \mu_{2}^{2}}{3 k_{B} T}\right)\right] \\
& =A \sum_{m}\left(\frac{\bar{Z}}{\bar{R}^{6}}\right)_{m}\left[\alpha_{1} B_{12}(I, \alpha, \mu)+\mu_{1}^{2} B_{2}(\alpha, \mu)\right] \\
& Z_{m}=\frac{N_{A}}{V}\left(\frac{4 \pi}{3} \bar{R}_{m}^{3}-V_{m}\right), \quad V_{m}=\sum V_{\mathrm{atom}}, \\
& \bar{R}_{m}=R_{2}+(2 m-1) R_{1}, \quad R_{i}=\left(4 \pi K^{*} M_{i} / 3 \rho_{i} N_{\mathrm{A}}\right)^{1 / 3} .
\end{aligned}
$$

Here $Z_{m}$ is the effective number of molecules in the $m$-th solvation shell of the radius $\bar{R}_{m}$ surrounding a particle of a solute, defined as the ratio of the area of the shell to the cross-section of a solvent molecule; $A$ is a constant, a veraged over $\delta_{j}$ and introduced to account for nonadditivity of LDK potentials $\Phi_{j} ; I_{1 i}$ and $\alpha_{i}$ are the first ionization potentials and dynamic polarizabilities of molecules $(i=1,2)$; $V, V_{m}$ and $V_{\text {atom }}$ are molar, molecular, and atomic volumes; $R_{i}$ denotes the radius of the sphere occupied by a single molecule at a given temperature $T$; and $K^{*}$ is the packing coefficient.

In agreement with (1) it is natural to assume that any molecular property ( $\mu$ in our case) of a substance changes during the gas-liquid phase transition proportionally to $\left\langle U_{12}\right\rangle_{\tau}$. For the first coordination shell $\left(m=1, K^{*}=0.74\right)$ in a binary system this fact can be expressed as

$$
\mu_{\text {ind }} \sim B_{12}(I, \alpha, \mu) Z_{1} \alpha_{1} \bar{R}_{1}^{-6}=B_{12} Z_{\alpha}
$$

for the solution with $\mu_{2} \neq 0$ and $\mu_{1}=0$, and 


$$
\mu_{\text {ind }} \sim B_{12} Z_{\alpha}+B_{2} Z_{\mu}
$$

for the solution with $\mu_{1} \neq 0$ and $\mu_{2} \neq 0$.

Our earlier analysis [6] of essentially all the a vailable literature data on dipole moments of organic molecules with a single substituent measured both in gaseous and liquid phases showed that for a polar substance dissolved in a series of similar apolar solvents the correlation (6) holds reasonably well in the covered range of $Z_{1}$ and $\alpha_{1}$

$$
\mu_{2},\left|\mu_{\mathrm{g} 2}-\mu_{2}\right| \approx \text { const }+B_{12} Z_{\alpha} .
$$

Experiments on dielectric properties of analogous solutions with solutes containing several substituents, such as 1-methoxy-2-nitrobenzene and $N, N$ dimethyl-2-nitroaniline, as well as those which exhibit intramolecular H-bonding, such as 2-nitrophenol and 2-hydroxybenzaldehyde, allowed us to extend the correlation to more complex substances. Meanwhile binary mixtures $\left(\mu_{1}=0\right)$ with solutes that show specific interactions do not conform to (8). It is not a drawback of the model potentials used in (5), but arises rather because of the approximate character of the expression for the total potential energy of a condensate molecule, which accounts only for the long-range forces.

Comparing the outcomes of using various models of dipole polarization applied to the problem of determinating gaseous phase electric moments of molecules in a condensed phase, we notice that the approximations involving the concepts of local (vs. Heaviside type) dielectric permittivity of a medium, reactive field and bulk molecular polarization are superior to the others. It seems valuable to complement this observation with the qualitative analysis of the role of the universal interparticle interactions in such predictions. Moreover, another simple way to estimate gaseous phase dipole moments follows from the experimentally tested expression (6). That is, mentally reducing the interaction energy of solvent and solute molecules (under the assumption that their structural and physical properties do not vary), we effectively transform a liquid into a gas. Thus, extrapolation of the functional dependence of (8) to zero argument $Z_{\alpha}$ can be considered to be a method to obtain $\mu_{\mathrm{g} 2}$.

\section{Dipole polarization of dilute solutions of polar substances in apolar solvents}

We measured dielectric properties of a series of dilute solutions with concentrations which satisfy relations (3). The technique and equipment are described elsewhere [7]. The solvents were of similar nature $\left(I_{11}, \alpha=\right.$ const), namely, paraffins, cycloparaffins and their halogen derivatives, cyclic ethers and thioethers, alkylbenzenes and some inorganic compounds. The physical characteristics of the solvents are given in Table I, where $\sigma$ is the scaling factor of the Lennard-Jones potential, $\omega$ denotes the Pitzer parameter [8] indicating how much aspherical a molecule is, $\bar{\alpha}$ is the average static polarizability, $\gamma_{\mathrm{c}}^{2}$ squared is molecular anisotropy of a condensate [9], and $I_{1}$ is the first ionization potential of a molecule [10]. Substances of the following classes were used as solutes: oxygen, nitrogen and 
TABLE I

The properties of the apolar solvents.

\begin{tabular}{c|l|c|c|c|c|r}
\hline \hline No. & \multicolumn{1}{|c|}{ Solvent } & $\sigma[\AA]$ & $\omega$ & $\bar{\alpha}\left[\AA^{3}\right]$ & $\gamma_{c}^{2}\left[\AA^{6}\right]$ & $I[\mathrm{eV}]$ \\
\hline 1 & $\mathrm{CH}_{3}\left(\mathrm{CH}_{2}\right)_{5} \mathrm{CH}_{3}$ & 5.561 & 0.351 & 13.67 & 6.1 & 10.08 \\
2 & $\mathrm{CH}_{3}\left(\mathrm{CH}_{2}\right)_{4} \mathrm{CH}_{3}$ & 4.424 & 0.296 & 11.81 & 4.7 & 10.18 \\
3 & $\mathrm{C}_{6} \mathrm{H}_{12}$ & 5.193 & 0.213 & 10.89 & 4.6 & 9.83 \\
4 & $\mathrm{CCl}_{4}$ & 5.054 & 0.194 & 11.15 & - & 11.47 \\
5 & $\mathrm{C}_{4} \mathrm{H}_{8} \mathrm{O}_{2}$ & 4.737 & 0.288 & 10.17 & 2.8 & 9.13 \\
6 & $\mathrm{C}_{6} \mathrm{H}_{6}$ & 4.917 & 0.212 & 10.54 & 30.5 & 9.25 \\
7 & $\mathrm{CS}_{2}$ & 4.329 & 0.115 & 8.44 & 76.1 & 10.07
\end{tabular}

TABLE II

The relationship between the magnitudes of dipole moments measured in a gaseous phase and in apolar solvents.

\begin{tabular}{|c|c|c|c|c|c|c|c|c|}
\hline \multirow[t]{3}{*}{ No. } & \multirow[t]{3}{*}{ Substance } & \multirow{2}{*}{\multicolumn{3}{|c|}{$\Delta \mu_{k}^{\mathrm{e}}$}} & \multirow{2}{*}{\multicolumn{4}{|c|}{$\frac{\text { Solvent }}{\mathrm{CH}_{3}\left(\mathrm{CH}_{2}\right)_{5} \mathrm{CH}_{3}}$}} \\
\hline & & & & & & & & \\
\hline & & $\Delta \mu_{1}^{\mathrm{e}}$ & $\Delta \mu_{2}^{\mathrm{e}}$ & $\Delta \mu_{3}^{e}$ & $\bar{Z}$ & $\Delta \mu_{1}$, & $\Delta \mu_{2}$ & $\dot{\Delta}_{\mu 3}$ \\
\hline 1 & $\mathrm{C}_{6} \mathrm{H}_{5} \mathrm{NH}_{2}$ & .0336 & .0102 & .0004 & 538 & .0384 & .0143 & .0032 \\
\hline 2 & $\mathrm{CH}_{3} \mathrm{COCH}_{3}$ & .0418 & .0104 & .0005 & - & - & - & - \\
\hline 3 & $\mathrm{CH}_{3} \mathrm{CN}$ & .0513 & .0136 & .0006 & - & - & - & - \\
\hline $4^{*}$ & $\mathrm{C}_{6} \mathrm{H}_{5} . \mathrm{OH}$ & .0368 & .0100 & - & - & - & - & - \\
\hline 5 & $\mathrm{C}_{6} \mathrm{H}_{5} \mathrm{CN}$ & .0607 & .0166 & .0008 & 503 & .0758 & .0306 & .0061 \\
\hline 6 & $\mathrm{C}_{6} \mathrm{H}_{5} \mathrm{Br}$ & .0327 & .0226 & .0003 & - & - & - & - \\
\hline 7 & $\left(\mathrm{CH}_{3}\right)_{3} \mathrm{CCl}$ & .0378 & .0104 & .0003 & 496 & .0507 & .0173 & .0038 \\
\hline $8^{*}$ & $\mathrm{C}_{6} \mathrm{H}_{5} \mathrm{CH}_{2} \mathrm{CH}_{2} \mathrm{C}_{6} \mathrm{H}_{5}$ & .0386 & .0253 & - & - & - & - & - \\
\hline 9 & $\mathrm{ClCH}_{2} \mathrm{CH}_{2} \mathrm{Cl}$ & .0359 & .0087 & .0004 & 572 & .0468 & .0163 & .0039 \\
\hline $10^{*}$ & $p-\mathrm{HOC}_{6} \mathrm{H}_{4} \mathrm{CH}_{2} \mathrm{NO}_{2}$ & .0788 & .0327 & - & 510 & .1014 & .0441 & .0053 \\
\hline 11 & $\mathrm{C}_{6} \mathrm{H}_{5} \mathrm{NO}_{2}$ & .0528 & .0151 & .0006 & 510 & .0644 & .0263 & .0054 \\
\hline $12^{*}$ & $p-\left(\mathrm{CH}_{3}\right)_{2} \mathrm{NC}_{6} \mathrm{H}_{4} \mathrm{NO}_{2}$ & .0933 & .0357 & - & 479 & .1174 & .0619 & .0072 \\
\hline 13 & $\mathrm{C}_{6} \mathrm{H}_{5} \mathrm{CH}_{2} \mathrm{NO}_{2}$ & .0684 & .0173 & - & 480 & .0824 & .0290 & .0053 \\
\hline $14^{*}$ & $o-\mathrm{HOC}_{6} \mathrm{H}_{4} \mathrm{NO}_{2}$ & .0381 & .0111 & - & 540 & .0503 & .0183 & .0035 \\
\hline 15 & $\mathrm{C}_{5} \mathrm{H}_{5} \mathrm{~N}$ & .0429 & .0122 & .0006 & 526 & .0553 & .0218 & .0043 \\
\hline $16^{*}$ & $\left(\mathrm{CH}_{3}\right)_{2} \mathrm{CHCN}$ & .0563 & .0191 & - & - & - & - & - \\
\hline 17 & $\mathrm{CH}_{3} \mathrm{CH}_{2} \mathrm{CH}_{2} \mathrm{Cl}$ & .0338 & .0067 & .0003 & - & - & - & - \\
\hline 18 & $\mathrm{C}_{6} \mathrm{H}_{5} \mathrm{COOH}$ & .0456 & .0116 & .0004 & 502 & .0567 & .0221 & .0040 \\
\hline 19 & $\mathrm{CHBr}_{2} \mathrm{CHBr}_{2}$ & .0338 & .0072 & .0002 & - & - & - & - \\
\hline 20 & $\mathrm{CHCl}_{2} \mathrm{CHCl}_{2}$ & .0377 & .0091 & .0003 & - & - & - & - \\
\hline 21 & $\mathrm{CH}_{3} \mathrm{COSH}$ & .0416 & .0099 & .0007 & - & - & - & - \\
\hline $22^{*}$ & $\mathrm{C}_{6} \mathrm{H}_{5} \mathrm{SH}$ & .0307 & .0051 & - & - & - & - & - \\
\hline 23 & $\mathrm{C}_{6} \mathrm{H}_{5} \mathrm{OH}$ & .0380 & .0082 & .0003 & - & - & - & - \\
\hline 24 & $\mathrm{C}_{6} \mathrm{H}_{5} \mathrm{Cl}$ & .0416 & .0096 & .0004 & - & - & - & - \\
\hline $25^{*}$ & $\mathrm{CH}_{3}\left(\mathrm{CH}_{2}\right)_{8} \mathrm{CH}_{2} \mathrm{Cl}$ & .0423 & .0050 & - & - & - & - & - . \\
\hline 26 & $\mathrm{CHCl}_{3}$ & .0313 & .0067 & .0002 & 595 & .0388 & .0131 & .0031 \\
\hline
\end{tabular}


halogen derivatives of benzene, alkylbenzenes, paraffins and cycloparaffins; and ketones, nitriles and some heterocyclic compounds (Table II). The calculated values of gaseous phase dipole moments of solute molecules $\mu_{2 k}$ were obtained within the limits of the original Onsager model [11], $k=1$, its modification [12], $k=2$, and our approach [1], $k=3$. The results are shown in Table II and, in part, in Figs. 1 and 2. The coordinate axes of the plots are $\Delta \mu_{k}$ and $Z_{\alpha}$, where $Z=Z_{\alpha} \times 10^{-18},\left[Z_{\alpha}\right]=\left[\mathrm{cm}^{3}\right] ; \Delta \mu_{k}=\left|\mu_{\mathrm{g} 2}-\mu_{2 k}\right| / \mu_{\mathrm{g} 2}, \Delta \mu_{k}^{\mathrm{e}}=\left|\mu_{\mathrm{g} 2}-\mu_{2 k}^{\mathrm{e}}\right| / \mu_{\mathrm{g} 2} ;$ $\mu^{\mathrm{e}}$ denotes gas phase dipole moment of a solute molecule found by extrapolating the function $\Delta \mu_{k}\left(Z_{\alpha}\right)$ to zero argument $Z_{\alpha}=0$. In the cases when experimental gas phase dipole moments are not available corresponding $\mu_{23}^{\mathrm{e}}$ were used instead.

TABLE II (the extention) The relationship between the magnitudes of dipole moments measured in a gaseous phase and in apolar solvents.

\begin{tabular}{|c|c|c|c|c|c|c|c|c|c|c|c|c|}
\hline \multirow{3}{*}{ No. } & \multicolumn{12}{|c|}{ Solvents } \\
\hline & \multicolumn{4}{|c|}{$\mathrm{CH}_{3}\left(\mathrm{CH}_{2}\right)_{4} \mathrm{CH}_{3}$} & \multicolumn{4}{|c|}{$\mathrm{C}_{6} \mathrm{H}_{12}$} & \multicolumn{4}{|c|}{$\mathrm{CCl}_{4}$} \\
\hline & $Z$ & $\Delta \mu_{1}$ & $\Delta \mu_{2}$ & $\Delta \mu_{3}$ & $Z$ & $\Delta \mu_{1}$ & $\Delta \mu_{2}$ & $\Delta \mu_{3}$ & $\bar{Z}$ & $\Delta \mu_{1}$ & $\Delta \mu_{2}$ & $\Delta \mu_{3}$ \\
\hline 1 & 550 & .0478 & .0183 & .0038 & 684 & .0477 & .0185 & .0044 & 770 & .0410 & .0157 & .0045 \\
\hline 2 & 660 & .0537 & .0222 & .0055 & - & - & - & - & 648 & .0716 & .0313 & .0075 \\
\hline 3 & 703 & .0712 & .0285 & .0069 & 884 & .0808 & .0349 & .0088 & 996 & .0721 & .0317 & .0092 \\
\hline $4^{*}$ & - & - & - & - & 746 & .0408 & .0142 & .0038 & 734 & .0530 & .0207 & .0043 \\
\hline 5 & 525 & .0742 & .0321 & .0063 & 762 & .0803 & .0353 & .0085 & 728 & .0873 & .0399 & .0087 \\
\hline 6 & 521 & .0449 & .0252 & .0029 & 643 & .0440 & .0241 & .0035 & 718 & .0511 & .0280 & .0073 \\
\hline 7 & 510 & .0457 & .0173 & .0039 & 545 & .0523 & .0193 & .0042 & 706 & .0597 & .0243 & .0055 \\
\hline $8^{*}$ & - & - & - & - & 500 & .0497 & .0289 & .0036 & 555 & .0430 & .0343 & .0443 \\
\hline 9 & 595 & .0497 & .0174 & .0040 & 738 & .0505 & .0183 & .0051 & 961 & .0483 & .0183 & .0059 \\
\hline $10^{*}$ & 525 & .0983 & .0427 & .0053 & 590 & .1027 & .0445 & .0067 & 660 & .1057 & .0464 & .0075 \\
\hline 11 & 525 & .0697 & .0284 & .0057 & 650 & .0683 & .0293 & .0067 & 730 & .0816 & .0365 & .0084 \\
\hline $12^{*}$ & 515 & .1292 & .0636 & .0076 & 610 & .1274 & .0703 & .0096 & 673 & .1118 & .0663 & .0097 \\
\hline $13^{*}$ & 493 & .0804 & .0245 & .0051 & - & - & - & - & 684 & .1060 & .0405 & .0080 \\
\hline $14^{*}$ & $560^{\circ}$ & .0537 & .0221 & .0044 & 625 & .0469 & .0143 & .0034 & 840 & .0657 & .0173 & .0051 \\
\hline 15 & 587 & .0587 & .0224 & .0031 & 733 & .0603 & .0251 & .0061 & 885 & .0583 & .0236 & .0068 \\
\hline $16^{*}$ & 562 & .0734 & .0331 & .0066 & 702 & .0744 & .0339 & .0080 & 781 & .0939 & .0516 & .0107 \\
\hline 17 & 563 & .0430 & .0135 & .0027 & - & - & - & - & 785 & .0356 & .0113 & .0032 \\
\hline 18 & 515 & .0533 & .0263 & .0038 & 640 & .0616 & .0251 & .0051 & 714 & .0683 & .0630 & .0062 \\
\hline 19 & 508 & .0430 & .0125 & .0035 & 636 & .0413 & .0101 & .0029 & 710 & .0376 & .0375 & .0027 \\
\hline 20 & 493 & .0472 & .0153 & .0033 & 610 & .0492 & .0156 & .0041 & - & - & - & - \\
\hline 21 & - & - & - & - & 777 & .0563 & .0203 & .0058 & 870 & .0639 & .0261 & .0063 \\
\hline $22^{*}$ & - & - & - & - & 654 & .0396 & .0103 & .0027 & 730 & .0323 & .0051 & .0024 \\
\hline 23 & - & - & - & - & 807 & .0500 & .0179 & .0048 & 787 & .0458 & .0125 & .0044 \\
\hline 24 & 524 & .0518 & .0169 & .0038 & 644 & .0522 & .0187 & .0045 & 734 & .0461 & .0135 & .0042 \\
\hline $25^{*}$ & 358 & .0500 & .0201 & .0027 & 440 & .0513 & .0227 & .0035 & 461 & .0463 & .0161 & .0027 \\
\hline 26 & 620 & .0426 & .0143 & .0033 & 723 & .0426 & .0163 & .0038 & 810 & .0359 & .0128 & .0039 \\
\hline
\end{tabular}


TABLE II (the extention) The relationship between the magnitudes of dipole moments measured in a gaseous phase and in apolar solvents.

\begin{tabular}{|c|c|c|c|c|c|c|c|c|c|c|c|c|}
\hline \multirow[t]{3}{*}{ No. } & \multicolumn{12}{|c|}{ Solvents } \\
\hline & \multicolumn{4}{|c|}{$\mathrm{C}_{4} \mathrm{H}_{8} \mathrm{O}_{2}$} & \multicolumn{4}{|c|}{$\mathrm{C}_{6} \mathrm{H}_{6}$} & \multicolumn{4}{|c|}{$\mathrm{CS}_{2}$} \\
\hline & $Z$ & $\Delta \mu_{1}$ & $\overline{\Delta \mu_{2}}$ & $\Delta \mu_{3}$ & $Z$ & $\Delta \mu_{1}$ & $\Delta \mu_{2}$ & $\Delta \mu_{3}$ & $\bar{Z}$ & $\Delta \mu_{1}$ & $\Delta \mu_{2}$ & $\Delta \mu_{3}$ \\
\hline 1 & 785 & .0564 & .0221 & .0052 & 867 & .0525 & .0223 & .0055 & 1409 & .0563 & .0261 & .0084 \\
\hline 2 & - & - & - & - & 945 & .0653 & .0299 & .0079 & 1653 & .0769 & .0383 & .0129 \\
\hline 3 & 993 & .0957 & .0426 & .0106 & 999 & .0739 & .0347 & .0101 & 1872 & .1023 & .0533 & .0173 \\
\hline $4^{*}$ & 746 & .0408 & .0142 & .0038 & 822 & .0693 & .0275 & .0057 & 1330 & .0578 & .0266 & .0072 \\
\hline 5 & 762 & .0803 & .0353 & .0085 & 813 & .0921 & .0423 & .0096 & 1485 & .1053 & .0558 & .0167 \\
\hline 6 & - & - & - & - & 804 & .0407 & .0223 & .0045 & 1354 & - & - & - \\
\hline 7 & 715 & .0483 & .0199 & .0052 & 790 & .0597 & .0246 & .0064 & 1280 & .0641 & .0293 & .0093 \\
\hline $8^{*}$ & 560 & .0602 & .0344 & .0045 & 618 & .0577 & .0328 & .0047 & 982 & .0603 & .0329 & .0084 \\
\hline 9 & 985 & .0597 & .0249 & .0068 & 931 & .0505 & .0192 & .0062 & 1562 & .0649 & .0297 & .0093 \\
\hline $10^{*}$ & 670 & .0844 & .0333 & .0064 & 737 & .1036 & .0437 & .0077 & 1190 & .1407 & .0613 & .0141 \\
\hline 11 & 740 & .0799 & .0343 & .0079 & 815 & .0703 & .0316 & .0080 & 1320 & .0924 & .0466 & .0135 \\
\hline $12^{*}$ & 681 & .1163 & .0677 & .0102 & 759 & .1453 & .0861 & .0124 & 1223 & .1566 & .1027 & .0947 \\
\hline $13^{*}$ & - & - & - & - & 760 & .0843 & .0296 & .0075 & 1380 & .1292 & .0512 & .0448 \\
\hline $14^{*}$ & 865 & .0613 & .0273 & .0059 & 785 & .0655 & .0267 & .0061 & 1400 & .0670 & .0334 & .0095 \\
\hline 15 & 837 & .0672 & .0301 & .0074 & 725 & .0670 & .0297 & .0063 & 1037 & .0648 & .0307 & .0081 \\
\hline $16^{*}$ & 796 & .0681 & .0347 & .0087 & 875 & .0742 & .0369 & .0099 & 1434 & .1042 & .0617 & .0164 \\
\hline 17 & - & - & - & - & 880 & .0550 & .0205 & .0045 & 1440 & .0582 & .0246 & .0066 \\
\hline 18 & 726 & .0523 & .0222 & .0049 & 800 & .0537 & .0246 & .0057 & 1296 & .0879 & .0413 & .0101 \\
\hline 19 & - & - & - & - & 762 & .0555 & .0207 & .0047 & 1230 & .0561 & .0207 & .0062 \\
\hline 20 & - & - & - & - & 794 & .0633 & .0231 & .0057 & 1284 & .0637 & .0261 & .0083 \\
\hline 21 & 890 & .0480 & .0147 & .0055 & 976 & .0697 & .0315 & .0075 & 1615 & .0785 & 0333 & .0117 \\
\hline $22^{*}$ & 743 & .0513 & .0177 & .0039 & - . & - & - & - & 1328 & .0496 & .0155 & .0056 \\
\hline 23 & 797 & .0603 & .0251 & .0054 & 880 & .0584 & .0223 & .0052 & 1446 & .0638 & .0249 & .0085 \\
\hline 24 & 740 & .0713 & .0331 & .0061 & 810 & .0657 & .0224 & .0059 & 1335 & .0682 & .0280 & .0090 \\
\hline $25^{*}$ & 462 & .0619 & .0285 & .0042 & 510 & .0618 & .0313 & .0045 & - & - & - & - \\
\hline 26 & 824 & .0381 & .0142 & .0040 & 909 & .0509 & .0191 & .0051 & - & - & - & - \\
\hline
\end{tabular}

Interpreting the experimental data and theoretical results, we notice the following. First, Eq. (8) for the function $\Delta \mu_{k}\left(Z_{\alpha}\right)$ holds in a substantial range of dipole moments of solute molecules: from $\mu_{23}=1.2 \mathrm{D}$ for chloroform to $\mu_{23}=$ $7.17 \mathrm{D}$ for $N, N$-dimethyl-4-nitroaniline, independent of the model for the internal field. Second, pairwise interactions of solute molecules, including those with several polar substituents $\left(\mathrm{C}_{7} \mathrm{H}_{7} \mathrm{O}_{3} \mathrm{~N}, \mathrm{C}_{8} \mathrm{H}_{10} \mathrm{~N}_{2} \mathrm{O}_{2}\right)$ and intermolecular hydrogen bonds (o-nitrophenol, 2-hydroxybenzaldehyde), with the surrounding particles of a solvent are clearly anisotropic for a wide range of structural and polarizational characteristics of solvent and solute molecules. Thus, for solvents the Pitzer asymmetry parameter $\omega$ varies from 0.115 for carbon sulfide to 0.361 for heptane, while the molecular anisotropy $\gamma_{\mathrm{c}}^{2}$ changes from $2.83 \AA^{6}$ for $\mathrm{C}_{4} \mathrm{H}_{8} \mathrm{O}_{2}$ to $76.13 \AA^{6}$ for carbon sulfide. The corresponding numbers for solutes are $\omega=0.190$ 


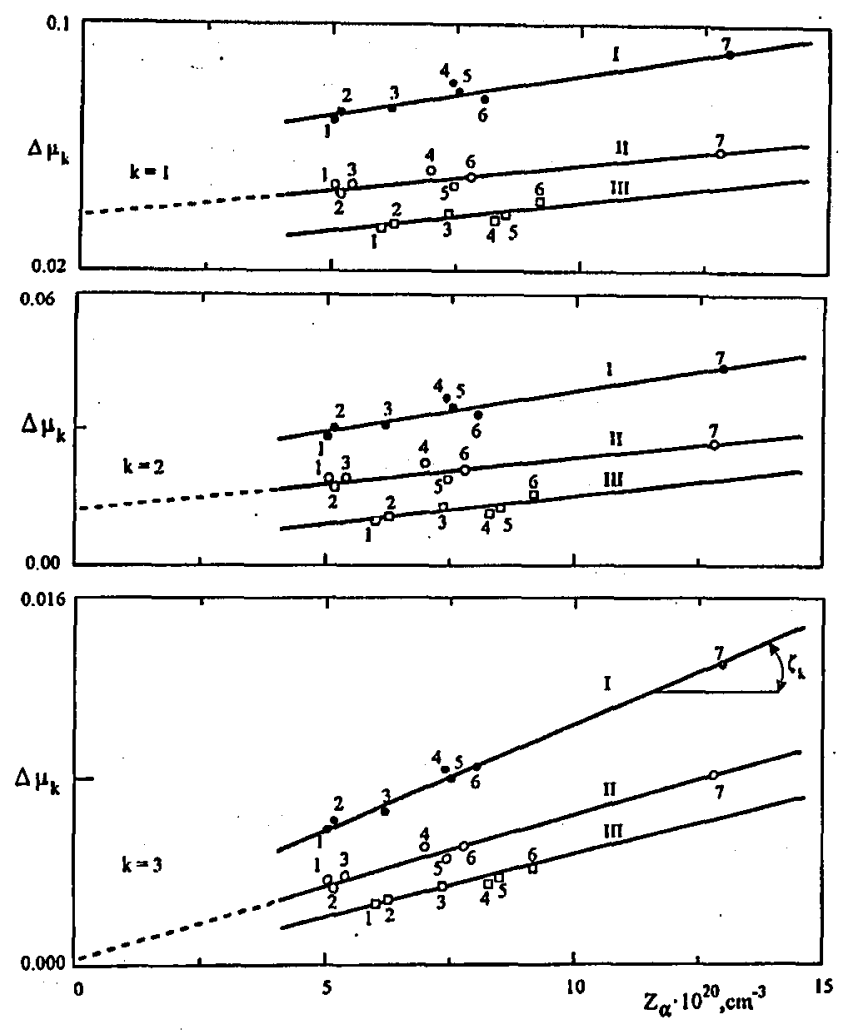

Fig. 1. The dependence of $Z_{\alpha}$ on $\Delta \mu_{k}$ for nitrobenzene (I), tert-butyl chloride (II) and chloroform (III) dissolved in nonpolar solvent (the points are numbers according to Table I) within the limits of the original Onsager model [11] $-k=1$; modification of the Onsager model [12] $-k=2$; and our approach [1] $-k=3$.

(2-methyl-2-chloropropane) and $\omega=0.440\left(\mathrm{C}_{6} \mathrm{H}_{5} \mathrm{OH}\right) ; \gamma_{\mathrm{c}}^{2}=2.6 \AA^{6}$ (acetone) and $\gamma_{\mathrm{c}}^{2}=200.0 \AA^{6}\left(\mathrm{C}_{6} \mathrm{H}_{5} \mathrm{NO}_{2}\right)$. A minor scatter of $\Delta \mu_{k}$ values is observed for solutions of practically all substances in tetrachloromethane, dioxane and benzene. This must have arisen from the deviations of the true ionization potentials of these compounds from the statistically averaged value $I_{11}=10.05 \mathrm{eV}$ (Table I). The dependence of the derivative $\xi_{k}$ of the function $\Delta \mu_{k}\left(Z_{\alpha}\right)$, which is directly related to the coefficient $B_{12}\left(I_{1}, \alpha, \mu\right)$ in (6), on the magnitudes of the dipole moments of solutes supports the correlation (5) between the magnitudes of dipole moments of the solutes and the energies of solute-solvent pairwise interactions. For example, the derivatives of $\Delta \mu_{k}$ calculated according to [11] exhibit consistent behavior changing $\operatorname{tg} \xi$ from $5 \times 10^{-3}$ for the $N, N$-dimethyl-4-nitroaniline in polar solvents to $8 \times 10^{-4}$ for the chloroform in non-polar solvents, as shown in Figs. 1 and 3, and where the derivative corresponds to the units of the axes. For the majority of solutes dissolved in $\mathrm{CCl}_{4}$ and $\mathrm{C}_{4} \mathrm{H}_{8} \mathrm{O}_{2}$; which usually do not follow general trends, 


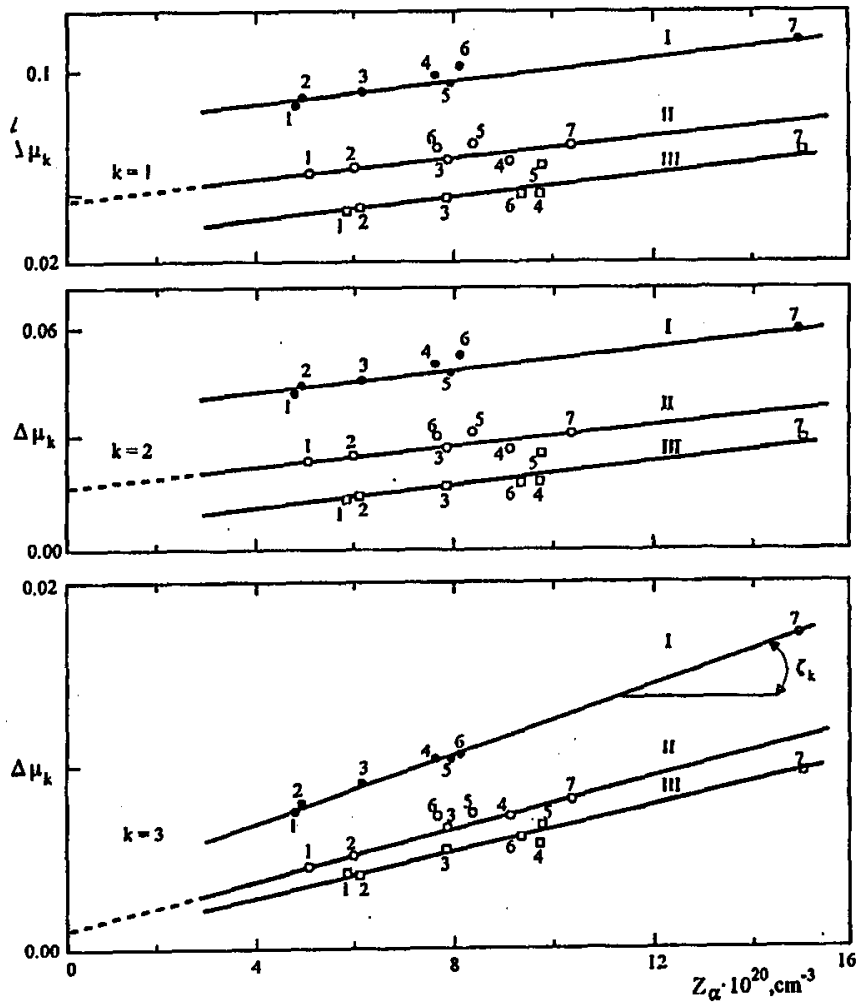

Fig. 2. The dependence of $Z_{\alpha}$ on $\Delta \mu_{k}$ for benzonitryl (I), pyridine (II) and 1,2-dichlorethane (III) dissolved in nonpolar solvent (the points are numbers according to Table I) within the limits of the original Onsager model [11] $-k=1$; modification of the Onsager model [12] $-k=2$; and our approach [1] $-k=3$.

our studies of the "dipole-induced dipole" and dispersive contributions to the total energy of interparticle solvent-solute interactions (5) argue against the existence of hydrogen bonded complexes between these solvents and the solutes containing $\mathrm{A}-\mathrm{H}$ groups, as well as against the importance of any coupling of group dipole moments in dilute solutions of these solvents [13]. It appears that $\Delta \mu_{k}$ of $\mathrm{CHCl}_{3}, \mathrm{C}_{6} \mathrm{H}_{5} \mathrm{SH}$, $\mathrm{C}_{6} \mathrm{H}_{5} \mathrm{OH}, \mathrm{CH}_{3} \mathrm{COSH}$ and $\mathrm{C}_{6} \mathrm{H}_{5} \mathrm{NH}_{2}$, which are apt to form hydrogen bonds with solvent molecules, do obey (6). This shows that the influence of specific interactions (e.g. H-bonding) on the electric properties of these substances is greater than the influence of an isotropic medium.

Differences in the models of dipole polarization of a condensate are reflected in the variations of the extrapolated gaseous phase dipole moments of solutes. First of all, the Heaviside step function, originally used by Onsager to describe the cavity-continuum border region, has been substituted for the exponential [12] and oscillating [2] functions. As a result the discrepancy between $\mu_{\mathrm{g} 2}$ and $\mu_{2}$ de- 
creased. Consistently incorporating the notions of high and low frequency molecular polarizations induced by the reactive field into the model [1] and regarding the Onsager cavity as a sphere containing a polarizable medium and a freely rotating rigid dipole in its center we were able to model the particle-environment electrostatic interaction energy more accurately. These new features lead to notable changes in the behavior of the function $\Delta \mu_{k}\left(Z_{\alpha}\right)$, as shown in Table II, and Figs. 1 and 2. Namely, $\Delta \mu_{1}>\Delta \mu_{2}>\Delta \mu_{3}$ and $\operatorname{tg} \xi_{1}>\operatorname{tg} \xi_{2}>\operatorname{tg} \xi_{3}$ for the ranges of polarizational, structural and physical-chemical properties of studied solutions. And finally, the graphical illustrations presented here convince us that extrapolation of the solute dipole moments $\mu_{2}^{\mathrm{e}}$ to $Z_{\alpha} \rightarrow 0$ can give good predictions for the gaseous phase dipole moments only within the limits of the approach developed in [1]. All other approaches result in a substantial repulsive interaction energy $\left(U_{\text {sum }}>0\right)$ remaining in the limit $Z_{\alpha}=0$ and larger errors in the predicted dipole moments $\Delta \mu_{1}^{\mathrm{e}}>\Delta \mu_{2}^{\mathrm{e}}>\Delta \mu_{3}^{\mathrm{e}}$. This result becomes particularly important when extrapolation is the only means to determine $\mu_{\mathrm{g} 2}^{\mathrm{e}}$, for example in the cases of limitedly soluble substances and those which cannot be transformed into a gas. Thus, model description of dipole polarization of dilute solutions of polar substances in apolar solvents presented earlier [1] agrees sufficiently well with the proposed approximate expression (5) for the energy of nonspecific intermolecular interactions given in terms of pairwise London-Falkenhagen-Debye potentials.

\section{Dipole polarization of dilute solution of polar compounds in polar solvents}

Next we studied corresponding characteristics of systems composed of polar solutes and polar solvents. According to Eqs. (5), (7) the induced contribution to the molecular dipole moment of a substance dissolved in a series of polar solvents with similar values of $I_{11}$ and $\alpha_{1}$ at fixed temperature is proportional to the solvent parameter $Z_{\mu}\left(Z_{\mu} \gg Z_{\alpha},\left[Z_{\mu}\right]=\left[e \mathrm{~cm}^{-4}\right]\right.$, where $e$ is the electronic charge in CGS units). The solvents that were used for our measurements were nitrile, sulfur, halogen, and nitrogen derivatives of alkanes (Table III).

TABLE III

The properties of the polar solvents.

\begin{tabular}{c|l|c|c|c|c|c|c}
\hline \hline No. & \multicolumn{1}{|c|}{ Solvent } & $\sigma[\AA]$ & $\omega$ & $\bar{\alpha}\left[\AA^{3}\right]$ & $\gamma_{\mathrm{c}}^{2}\left[\AA^{6}\right]$ & $I_{1}[\mathrm{eV}]$ & $\mu_{\mathrm{g}}[\mathrm{D}]$ \\
\hline 1 & $\left(\mathrm{C}_{2} \mathrm{H}_{5}\right)_{3} \mathrm{~N}$ & 6.090 & 0.329 & 19.52 & 2.30 & 7.50 & 0.66 \\
2 & $\mathrm{CH}_{3} \mathrm{CH}_{2} \mathrm{NHCH}_{2} \mathrm{CH}_{3}$ & 5.555 & 0.299 & 19.05 & 2.27 & 8.51 & 0.92 \\
3 & $\mathrm{CH}_{3} \mathrm{CH}_{2} \mathrm{OCH}_{2} \mathrm{CH}_{3}$ & 4.585 & 0.192 & 21.64 & 2.62 & 10.00 & 1.29 \\
4 & $\mathrm{CH}_{3} \mathrm{COOCH}_{3}$ & 5.165 & 0.324 & 20.72 & 7.41 & 10.27 & 1.71 \\
5 & $\left(\mathrm{CH}_{3}\right)_{3} \mathrm{CCl}$ & 5.250 & 0.301 & 27.90 & 6.52 & 10.65 & 1.96 \\
6 & $\mathrm{HCON}\left(\mathrm{CH}_{3}\right)_{2}$ & - & - & 28.20 & - & 9.12 & - \\
7 & $\mathrm{CH}_{3} \mathrm{NO}_{2}$ & 4.912 & 0.346 & 19.65 & 3.95 & 11.10 & 3.46 \\
8 & $\left(\mathrm{CH}_{3}\right)_{2} \mathrm{SO}$ & - & - & 26.42 & 1.58 & 8.85 & - \\
9 & $\mathrm{CH}_{3} \mathrm{CN}$ & 5.251 & 0.321 & 19.13 & 4.43 & 12.22 & 3.91
\end{tabular}


TABLE IV

The relationship between magnitudes of dipole moments measured in a gaseous phase and in polar solvents.

\begin{tabular}{l|l|c|c|c|c|c|c|c}
\hline \hline \multirow{2}{*}{ No. } & \multicolumn{3}{|c|}{ Substance } & \multicolumn{3}{c|}{$\Delta \mu_{k}^{\mathrm{e}}$} & \multicolumn{4}{c}{ Solvent } \\
\cline { 3 - 8 } & & \multicolumn{3}{c}{$\left(\mathrm{C}_{2} \mathrm{H}_{5}\right)_{3} \mathrm{~N}$} \\
\cline { 3 - 8 } & & $\Delta \mu_{1}^{\mathrm{e}}$ & $\Delta \mu_{2}^{\mathrm{e}}$ & $\Delta \mu_{3}^{\mathrm{e}}$ & $Z$ & $\Delta \mu_{1}$ & $\Delta \mu_{2}$ & $\Delta \mu_{3}$ \\
\hline 1 & $\mathrm{C}_{6} \mathrm{H}_{5} \mathrm{Cl}$ & .0347 & .0103 & .0048 & 1.9 & .0562 & .0200 & .0092 \\
$2^{*}$ & $\mathrm{C}_{6} \mathrm{H}_{5} \mathrm{COOH}$ & .0372 & .0133 & - & 1.4 & .0701 & .0434 & .0074 \\
$3^{*}$ & $0-\mathrm{HOC}_{6} \mathrm{H}_{4} \mathrm{NO}_{2}$ & .0452 & .0174 & - & 2.1 & .0896 & .0409 & .0070 \\
$4^{*}$ & $\mathrm{C}_{6} \mathrm{H}_{5} \mathrm{NO}_{2}$ & .0615 & .0185 & - & 1.9 & .0286 & .0073 & .0062 \\
$5^{*}$ & $\mathrm{C}_{6} \mathrm{H}_{5} \mathrm{CN}^{*}$ & .0726 & .0233 & - & 2.0 & .1093 & .0498 & .0122 \\
$6^{*}$ & $p-\mathrm{HOC}_{6} \mathrm{H}_{4} \mathrm{CH}_{2} \mathrm{NO}_{2}$ & .0921 & .0277 & - & 1.9 & .1187 & .0486 & .0103 \\
$7^{*}$ & $p-\left(\mathrm{CH}_{3}\right)_{2} \mathrm{NC}_{6} \mathrm{H}_{4} \mathrm{NO}_{2}$ & .1217 & .0404 & - & 1.6 & .0867 & .0285 & .0069 \\
\hline${ }^{*} \Delta \mu_{k}=\left|\mu_{23}^{\mathrm{e}}-\mu_{2 k}\right|\left(\mu_{23}^{\mathrm{e}}\right)^{-1} ; \Delta \mu_{k}^{\mathrm{e}}=\left|\mu_{23}^{\mathrm{e}}-\mu_{2 k}^{\mathrm{e}}\right|\left(\mu_{23}^{\mathrm{e}}\right)^{-1}$.
\end{tabular}

TABLE IV (the extention) The relationship between magnitudes of dipole moments measured in a gaseous phase and in polar solvents.

\begin{tabular}{l|c|c|c|c|c|c|c|c|c|c|c|c}
\hline \multirow{2}{*}{ No. } & \multicolumn{10}{c}{ Solvents } \\
\cline { 2 - 12 } & \multicolumn{3}{|c|}{$\mathrm{CH}_{3} \mathrm{CH}_{2} \mathrm{NHCH}_{2} \mathrm{CH}_{3}$} & \multicolumn{3}{c|}{$\mathrm{CH}_{3} \mathrm{CH}_{2} \mathrm{OCH}_{2} \mathrm{CH}_{3}$} & \multicolumn{4}{c}{$\mathrm{CH}_{3} \mathrm{COOCH}_{3}$} \\
\cline { 2 - 11 } & $Z$ & $\Delta \mu_{1}$ & $\Delta \mu_{2}$ & $\Delta \mu_{3}$ & $Z$ & $\Delta \mu_{1}$ & $\Delta \mu_{2}$ & $\Delta \mu_{3}$ & $Z$ & $\Delta \mu_{1}$ & $\Delta \mu_{2}$ & $\Delta \mu_{3}$ \\
\hline 1 & 10.6 & .0286 & .0165 & .0143 & 10.9 & .0594 & .0215 & .0136 & 34.4 & .0853 & .0355 & .0182 \\
$2^{*}$ & 7.2 & .0186 & .0057 & .0029 & 7.3 & .0486 & .0166 & .0060 & 22.8 & .0914 & .0426 & .0085 \\
$3^{*}$ & 11.7 & .0334 & .0147 & .0063 & 16.3 & .0840 & .0327 & .0082 & 39.5 & .1487 & .0646 & .0111 \\
$4^{*}$ & 10.6 & .1163 & .0220 & .0094 & 10.8 & .0913 & .0331 & .0089 & 34.2 & .1387 & .0685 & .0121 \\
$5^{*}$ & 10.6 & .0805 & .0487 & .0113 & 10.8 & .1128 & .0347 & .0089 & 34.2 & .2007 & .0835 & .0124 \\
$6^{*}$ & 10.2 & .1267 & .0347 & .0083 & 9.7 & .1453 & .0443 & .0096 & 33.2 & .2583 & .0843 & .0134 \\
$7^{*}$ & 9.2 & .2201 & .0751 & .0045 & 9.4 & .1762 & .0613 & .0062 & 29.4 & .2787 & .1022 & .0083 \\
\hline${ }^{*} \Delta \mu_{k}=\left|\mu_{23}^{\mathrm{e}}-\mu_{2 k}\right|\left(\mu_{23}^{\mathrm{e}}\right)^{-1} ; \Delta \mu_{k}^{\mathrm{e}}=\left|\mu_{23}^{\mathrm{e}}-\mu_{2 k}^{\mathrm{e}}\right|\left(\mu_{23}^{\mathrm{e}}\right)^{-1}$. & & &. &
\end{tabular}

TABLE IV (the extention) The relationship between magnitudes of dipole moments measured in a gaseous phase and in polar solvents.

\begin{tabular}{l|c|c|c|c|c|c|c|c|c|c|c|c}
\hline \multirow{2}{*}{ No. } & \multicolumn{10}{c|}{ Solvents } \\
\cline { 2 - 13 } & \multicolumn{4}{|c|}{$\left(\mathrm{CH}_{3}\right)_{3} \mathrm{CCl}$} & \multicolumn{4}{c|}{$\mathrm{HCON}\left(\mathrm{CH}_{3}\right)_{2}$} & \multicolumn{3}{c}{$\mathrm{CH}_{3} \mathrm{NO}_{2}$} \\
\cline { 2 - 13 } & $Z$ & $\Delta \mu_{1}$ & $\Delta \mu_{2}$ & $\Delta \mu_{3}$ & $Z$ & $\Delta \mu_{1}$ & $\Delta \mu_{2}$ & $\Delta \mu_{3}$ & $Z$ & $\Delta \mu_{1}$ & $\Delta \mu_{2}$ & $\Delta \mu_{3}$ \\
\hline 1 & 36.8 & .1121 & .0452 & .0182 & 181.5 & .2080 & .1450 & .0194 & 219.2 & .2267 & .1752 & .0090 \\
$2^{*}$ & 24.9 & .1067 & .0306 & .0073 & 120.8 & .2381 & .0995 & .0174 & 143.7 & .2560 & .1118 & .0195 \\
$3^{*}$ & 40.7 & .1245 & .0503 & .0102 & - & - & - & - & 245.2 & .4344 & .2436 & .0348 \\
$4^{*}$ & 34.2 & .1633 & .0753 & .0128 & 180.6 & .3667 & .2972 & .0305 & 217.9 & .3754 & .2374 & .0334 \\
$5^{*}$ & 36.6 & .1869 & .1039 & .0151 & 180.7 & .4561 & .2407 & .0382 & 218.1 & .4442 & .2439 & .0382 \\
$6^{*}$ & 35.6 & .2876 & .1046 & .0158 & 175.2 & .4826 & .2249 & .0339 & 211.1 & .5323 & .2944 & .0447 \\
$7^{*}$ & 50.2 & .3187 & .1307 & .0213 & 155.5 & .5022 & .2684 & .0412 & 185.8 & .5774 & .3080 & .0227 \\
\hline
\end{tabular}

${ }^{*} \Delta \mu_{k}=\left|\mu_{23}^{\mathrm{e}}-\mu_{2 k}\right|\left(\mu_{23}^{\mathrm{e}}\right)^{-1} ; \Delta \mu_{k}^{\mathrm{e}}=\left|\mu_{23}^{\mathrm{e}}-\mu_{2 k}^{\mathrm{e}}\right|\left(\mu_{23}^{\mathrm{e}}\right)^{-1}$. 
TABLE IV (the extention) The relationship between magnitudes of dipole moments measured in a gaseous phase and in polar solvents.

\begin{tabular}{l|c|c|c|c|c|c|c|c}
\hline \hline \multirow{2}{*}{ No. } & \multicolumn{8}{|c}{ Solvents } \\
\cline { 2 - 9 } & \multicolumn{3}{|c}{$\left(\mathrm{CH}_{3}\right)_{2} \mathrm{SO}$} & \multicolumn{4}{c}{$\mathrm{CH}_{3} \mathrm{CN}$} \\
\cline { 2 - 8 } & $Z$ & $\Delta \mu_{1}$ & $\Delta \mu_{2}$ & $\Delta \mu_{3}$ & $Z$ & $\Delta \mu_{1}$ & $\Delta \mu_{2}$ & $\Delta \mu_{3}$ \\
\hline 1 & 211.2 & 0.2135 & 0.1535 & 0.0463 & 223.8 & 0.2887 & 0.1842 & 0.0455 \\
$2^{*}$ & 140.2 & 0.3162 & 0.1426 & 0.0224 & 146.7 & 0.3268 & 0.1507 & 0.0301 \\
$3^{*}$ & 235.4 & 0.3931 & 0.2153 & 0.0319 & 252.1 & 0.3533 & 0.2197 & 0.0328 \\
$4^{*}$ & 210.0 & 0.4364 & 0.2231 & 0.0334 & 222.5 & 0.4566 & 0.2723 & 0.0367 \\
$5^{*}$ & 210.1 & 0.5008 & 0.2938 & 0.0408 & 222.7 & 0.4247 & 0.2451 & 0.0436 \\
$6^{*}$ & 203.8 & 0.5083 & 0.2507 & 0.0386 & 215.6 & 0.4941 & 0.2436 & 0.0381 \\
$7^{*}$ & 180.5 & 0.5562 & 0.3007 & 0.0208 & 190.3 & 0.4823 & 0.2873 & 0.0244 \\
\hline \multirow{2}{*}{$* \mu_{k}=\left|\mu_{23}^{\mathrm{e}}-\mu_{2 k}\right|\left(\mu_{23}^{\mathrm{e}}\right)^{-1} ; \Delta \mu_{k}^{\mathrm{e}}=\left|\mu_{23}^{\mathrm{e}}-\mu_{2 k}^{\mathrm{e}}\right|\left(\mu_{23}^{\mathrm{e}}\right)^{-1}}$. \\
\end{tabular}

The solutes were nitrile, oxygen, nitrogen and halogen derivatives of benzene (Table III). The technique and conditions of the experiments as well as the procedure for calculating $\Delta \mu_{k}, \Delta \mu_{k}^{\mathrm{e}}(k=1,2,3)$ were the same as those described above. The results summarized in Table IV and partially presented in Fig. 3 (in the coordinate system $\left.\Delta \mu_{k}\left(Z_{\alpha}\right)\right)$ allow us to state the following. The functions $\Delta \mu_{k}\left(Z_{\alpha}\right)$ and $\Delta \mu_{k}\left(Z_{\mu}\right)$ corresponding to apolar and polar media are qualitatively similar for the magnitudes of $Z_{\mu}$ up to $8 \times 10^{-8} e \mathrm{~cm}^{-4}$, i.e. for solutions with relatively small energies of dipole-dipole pairwise interactions of polar molecules. In agreement with Eqs. (1), (5) there exists a direct correlation between the magnitudes of interacting molecular electric moments and the induced contributions to $\mu_{2 \mathrm{c}}$. For example, $\Delta \mu_{k}$ values for almost all solutes calculated according to [11] reach maxima for solutions in acetonitrile. Their relative values increase in proportion to $\mu_{2}$ (Table IV, Fig. 3). For the magnitudes of $Z_{\mu}$ above $8 \times 10^{-8} e \mathrm{~cm}^{-4}$ the function $\Delta \mu_{k}\left(Z_{\mu}\right)$ deviates significantly from linearity. These values of $Z_{\mu}$ correspond to highly polar solvents $\left(\mu_{1 \mathrm{~g}}>3 \mathrm{D}\right)$. Large solute dipole moments $\mu_{2}$ lead to further increase in the curvature of $\Delta \mu_{k}\left(Z_{\mu}\right)$. This effect can be interpreted as a consequence of the developing locality (anisotropy) of electrostatic interparticle interactions. A nonlocal (isotropic, bulk) character of pairwise interactions can be attributed predominantly to dipole-induced dipole and dispersive forces, as established in the preceding section. The nonlinear behavior of $\Delta \mu_{k}\left(Z_{\mu}\right), Z_{\mu}>$ $8 \times 10^{-8} e \mathrm{~cm}^{-4}$, independent of polarizational and structural properties of interacting particles, confirms this conclusion. Finally, tabulated and plotted data for the "polar solute-polar solvent" systems scatter to a significantly greater extent than the data for the "polar solute-apolar solvent" systems. In most cases it is attributable to specific interparticle interactions, not considered in (5). For instance, 2-hydroxybenzaldehyde molecules dissolved in $\left(\mathrm{C}_{2} \mathrm{H}_{5}\right)_{3} \mathrm{~N}, \mathrm{CH}_{3} \mathrm{CH}_{2} \mathrm{NHCH}_{2} \mathrm{CH}_{3}$ and $\mathrm{ICON}\left(\mathrm{CII}_{3}\right)_{2}$ undergo dissociation of the intramolecular hydrogen bond with subsequent formation of intermolecular hydrogen bonds of the N... O-H type with solvent molecules. These interactions affect the calculated values of $\mu_{2}$ (Table IV). The above mentioned deviations from the bulk character of interparticle interaction, such as the formation of complexes due to local (anisotropic) dipole-dipole interactions, are especially typical of highly polar compounds with several func- 

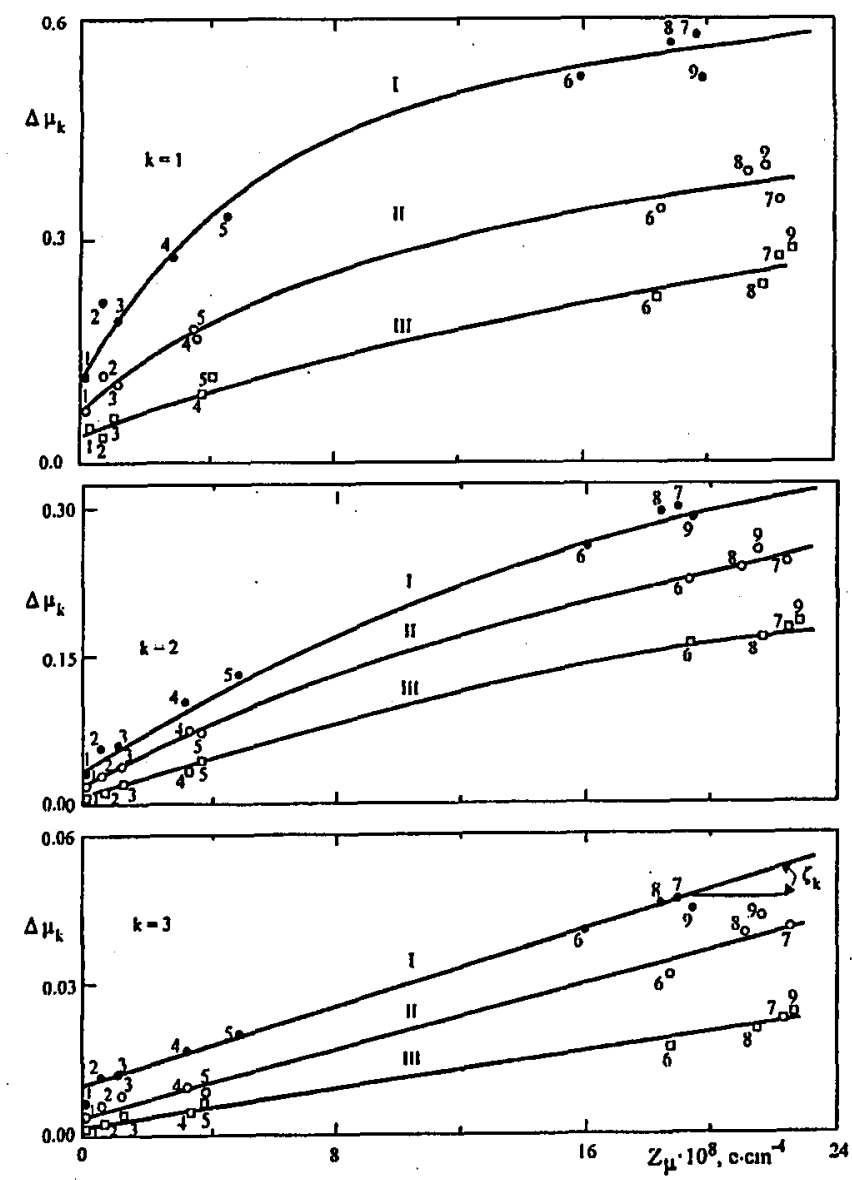

Fig. 3. The dependence of $Z_{\mu}$ on $\Delta \mu_{k}$ for $N, N$-dimethyl-4-nitroaniline (I), nitrobenzene (II) and chlorbenzene (III) dissolved in polar solvent (the points are numbers according to Table III) within the limits of the original Onsager model [11] $-k=1$; modification of the Onsager model [12] $-k=2$; and our approach [1] $-k=3$.

tional groups, $N, N$-dimethyl-4-nitroaniline and $\mathrm{C}_{6} \mathrm{H}_{5} \mathrm{CH}_{2} \mathrm{NO}_{2}$ being particular examples. Calculated $\Delta \mu_{k}\left(Z_{\mu}\right)$ values scatter noticeably for solutions of these substances in all polar solvents (Fig. 3, Table IV).

Two factors determine the quality of the model description of dipole polarization of condensates applied to the experimental dipole moment data for binary solutions of both types (polar-apolar and polar-polar). These are the internal field structure incorporated in the model and the manner in which the model accounts for the potential energy (5) of a molecule submerged in a solvent. Considering the influence of only nonspecific van-der-Waals interactions on the calculated gaseous phase dipole moments of polar molecules in a polar condensed systems of the sec- 
ond type, we give preference to the new model [1] as well. Calculated values of the parameters $\Delta \mu_{k}, \Delta \mu_{k}^{\mathrm{e}}$ and $\operatorname{tg} \xi_{k}$ of the function $\Delta \mu_{k}\left(Z_{\mu}\right)$ obey the inequalities $\Delta \mu_{3}, \Delta \mu_{3}^{\mathrm{e}}<\Delta \mu_{2}, \Delta \mu_{2}^{\mathrm{e}}<\Delta \mu_{1}, \Delta \mu_{1}^{\mathrm{e}}$ and $\operatorname{tg} \xi_{3}<\operatorname{tg} \xi_{2}<\operatorname{tg} \xi_{1}$ for the whole range of solute and solvent properties (Table IV, Fig. 3). In addition, as in the case of polar solutes and apolar solvents, the extrapolated values of $\mu_{2}^{\mathrm{e}}$ reach the experimental ones $\left(\mu_{\mathrm{g} 2}\right)$ only if the former are obtained by the model [1]. The predictions of other models of the internal field, e.g., from Refs. [11] and [12], extrapolated to $Z_{\mu} \rightarrow 0$ do not lead to zero interaction energy but rather to a positive one, which is unphysical and cannot represent the transition to the gaseous phase.

\section{Conclusions}

We based our investigation on dielectric properties measurements in dilute binary solutions having components whilch cover broad ranges of physical, chemical, structural, and polarizational properties. We were able to set up the limits for the description of the potential energy of interacting molecules (5) by pairwise London-Debye-Keesom potentials and tested several continuum models for the electric polarization of fluids. From expression (5) for the total energy of a molecule in a solution we used these models to estimate gaseous phase dipole moments of solvated molecules by extrapolating corresponding liquid phase values to zero energy of interaction of molecules with their environment. To recapitulate:

1. London-Debye-Keesom potentials have been used to the evaluation of the influence of pairwise (dispersive, inductive and dipole) molecular interactions on the polarization properties of a condensate. We have observed linear correlations between the electric molecular moments of solute molecules and the solvent parameters: $Z\left(Z=z_{1} a_{1} \bar{R}_{1}^{6}\right)$ for solvents having apolar particles with similar dimensions and ionization potentials, and $Z_{\mu}=z_{1} \mu_{1} \bar{R}_{1}^{6}\left(Z_{\mu}<8 \times 10^{8} \mathrm{e} \mathrm{cm}^{-4}\right)$ for solvents having polar particles with similar dimensions, ionization potentials and polarizabilities. The temperature is assumed to be constant. The correlations hold both for solutes which do not exhibit hydrogen bonding or other types of specific interactions and for solutes with intramolecular hydrogen bonds, if the solvents are not strong proton acceptors and cannot destroy these bonds. For solutions with $Z_{\mu}>8 \times 10^{8} \mathrm{e} \mathrm{cm}^{-4}$ we see deviations from linearity which tend to increase as the solution components become more polar.

2. Comparing the models of dipole polarization of condensates, we give preference to approaches which develop the concepts of reactive field and local dielectric permittivity and consider a condensate in a quasi-continuous fashion and introducing an oscillating function to describe a particle with its nearest surroundings, rather than regarding it as a continuum with the Heaviside type electric induction on the "Onsager cavity-structureless bulk" border. We have shown that this model is the most successful one when used to account for universal van-der-Waals interactions. It predicts gaseous phase dipole moments of solvated substances most accurately.

3. Simple extrapolation of the experimental data for dipole moments of substances dissolved in analogous apolar and polar solvents to vanishing solute-solvent interactions described within the limits of the model [1] allowed us to estimate 
gaseous phase dipole moments of thirty five organic compounds, most of which cannot be studied in the gas phase directly.

\section{References}

[1] N.I. Ivanov, V.V. Prezhdo, G.V. Tarasova, T.N. Kurskaya, O.V. Prezhdo, S.A. Tyurin, Zh. Fiz. Khim. 68, 159 (1994).

[2] S.A. Tyurin, M.V. Khashchina, Zh. Fiz. Khim. 61, 159 (1987).

[3] V.A. Gorodyski, N.G. Bahshiev, L.F. Kardashina, Spektroskopiya Vnutri i Mezhmolekularnykh Vzaimodeystviy (USSR) 1, 63 (1975).

[4] M.V. Vol'kenstein, M.A. El'iashevich, B.N. Stepanov, Kolebaniya Molekul, Vol. 2, Gos. Izdat. Tekhn. Literatury, Moskva 1949.

[5] A.E. Luckiy, S.N. Vragova, Zh. Fiz. Khim. 49, 2869 (1975).

[6] V.V. Prezhdo, I.P. Kraynov, Mezhmolekularnyie vzaimodeystvia i elektricheskie svoystva molekul, Osnova, Kharkov 1994.

[7] V.V. Prezhdo, M.V. Khashchina, V.A. Zamkov, Elektroopticheskie issledovaniya $v$ fizikie i khimii, Vyssha Shkola, Kharkov 1982.

[8] K.S. Pitzer, D.Z. Lipmann, R.F. Curl, C.M. Huggins, D.E. Peterson, J. Am. Chem. Soc. 18, 3433 (1955).

[9] A.N. Vereshchagin, Polarizuyemost' molekul, Nauka, Moskva 1982.

[10] F.I. Vilesov, Uspiekhi fotoniki (Leningrad) 1, 68 (1969).

[11] S. Filipczuk, G. Khanarian, J. Chem. Soc. Faraday Trans. 77, 477 (1981).

[12] H. Block, S.M. Walker, Chem. Phys. Lett. 19, 363 (1977).

[13] P. Suppan, Spectrochim. Acta A 28, 599 (1972). 